\title{
Assessment of Knowledge of Dental Staff at a Saudi Arabian University Regarding the Prophylaxis for Infective Endocarditis
}

\author{
Ahmed Bhayat", Fadi Jarab, Samir Mansuri, Mohammad Sami Ahmad and Mohamed Saad Mahrous \\ College of Dentistry, Taibah University, Al Madinah Al Munawwarah, Kingdom of Saudi Arabia
}

\begin{abstract}
The use of prophylactic antibiotics for the prevention of infective endocarditis following dental procedures has long been debated and there is still confusion regarding its efficacy. As a result, the prophylactic treatment varies considerably amongst different countries across the world and amongst different dental practitioners.

Aim: To evaluate the knowledge of dental staff regarding the guidelines for the prevention of infective endocarditis.

Methods: This was a cross sectional analytical study which included all staff members. A self administered questionnaire was used and responses were "graded" according to the American Heart Association (AHA) guidelines.

Results: The response rate was $87 \%(\mathrm{~N}=39)$ and $97 \%$ reported to use the AHA guidelines; $66 \%$ reported their knowledge was based on previous training and scientific journals. Of those cardiac conditions and dental procedures which required prophylaxis; $47 \%$ and $65 \%$ chose the correct option, respectively. Penicillin was prescribed as the drug of choice by the majority of respondents.
\end{abstract}

Conclusions: Although almost all staff reported the use of the AHA guidelines, many were not following them. The study emphasizes the need for continuous education and evaluation of this critical aspect of dentistry.

Keywords: Dental staff, Infective endocarditis, Knowledge, Prophylactic antibiotics.

\section{INTRODUCTION}

The use of prophylactic antibiotics (ABs) for the prevention of infective endocarditis (IE) following dental procedures has long been debated and there is still confusion regarding its efficacy [1]. As a result, prophylactic treatment varies considerably amongst different countries across the world [2] and the most common guidelines are from the American Heart Association (AHA), the British Society for Antimicrobial Chemotherapy (BSAC) and the National Institute for Health and Clinical Excellence (NICE) [2, 3]. These guidelines continue to be updated as new evidence is published and this impacts on the cardiac conditions that require prophylaxis and the different types of dental procedures that necessitate the need for ABs [2,3]. A review by the Canadian Pediatric Society [3] reported a wide variation between the different guidelines and an equally wide variation between the earlier and later versions of guidelines developed by the same authorizing body. These differences heighten the confusion amongst dental practitioners since they often contradict each other and as a result, many practitioners prescribe ABs unnecessarily or incorrectly $[4,5]$. Parrish et al. (2012) [6] concluded that AB coverage has modest preventive value in the prevention of IE following dental procedures but there needs to be standardized guidelines that are acceptable and practical. A study [7] reported that a patient

*Address correspondence to this author at the College of Dentistry, Taibah University, Al Madinah Al Munawwarah 43353, Kingdom of Saudi Arabia, Tel: 0593860175; E-mail: ahmedbhayat6@gmail.com with IE who did not receive $\mathrm{AB}$ coverage went on to develop severe complications following a dental procedure. The reason for not prescribing prophylactic ABs was as a result of the new updated guidelines and the interpretation of these guidelines by the clinician and physician. As a result, the author [7] stressed that clinicians need to be aware of and understand the modified guidelines and prescribe ABs appropriately or face dire consequences. In addition, many studies [8-10] have reported that dentists in private practice have limited and outdated knowledge regarding the prophylactic guidelines for the prevention of IE. Even where dentists had sufficient knowledge there was still confusion regarding certain dental procedures [10].

No study has been done in Saudi Arabia, either in the private sector or at an academic institution to determine the knowledge of the guidelines for the prevention of IE amongst dental staff.

This was the first study undertaken amongst staff members at Taibah University, College of Dentistry (TUCoD) in Saudi Arabia. As the university has employed staff members from different countries, it is expected that there may be a wide variation in the knowledge and practice of the prophylactic guidelines for the prevention of IE. It has been agreed that TUCoD will follow the updated AHA guidelines [11] and therefore, it was decided to determine the level of knowledge and practice of staff regarding these guidelines. Many studies [12-14] have concluded that there is a need for the improvement, updating and educating of clinicians regularly to ensure that they prescribe the most effective prophy- 
lactic regimens. Even within a dental school, Murrah et al. [15] showed a wide discrepancy between the prescription of prophylactic $\mathrm{ABs}$ and the knowledge of dental staff regarding the guidelines for IE.

The aim was to evaluate the knowledge of dental staff regarding the guidelines for the prevention of IE.

\section{SUBJECTS AND METHODS}

This was a cross sectional analytical study and all staff members (full time and part time) employed at TUCoD during the 2012 academic year were asked to participate. There were 45 staff members employed and each of them received a questionnaire together with a covering letter describing the rationale for the study.

The data were collected using a self administered questionnaire that was developed after a thorough literature review on the subject matter. It was pre-tested on medical staff members at Taibah University and after receiving feedback, it was modified slightly. It was in the English medium and consisted of 40 closed and 10 open ended questions. It was divided into 5 sections;

1. Demographics: Closed and open ended questions related to gender, age, specialty and years of experience.

2. General considerations: Open ended questions about referral practices and the choice of prophylactic guideline used when prescribing $\mathrm{ABs}$ for the prevention of IE.

3. Cardiac conditions for prescribing prophylactic antibiotics: This section addressed the prescription of $\mathrm{ABs}$ for the prevention of IE from 15 cardiac conditions. The respondents selected one of three options: "Yes, I prescribe", "No, I do not prescribe" and "I don't know". The scores were added and averages were obtained. The results were compared and assessed against the guidelines they reported to be following.

4. Dental procedures for prescribing prophylactic antibiotics: this section listed 18 common dental procedures and respondents were asked whether they would prescribe ABs or not for the prevention of IE. They could choose one of the following options: "Yes, I prescribe", "No, I do not prescribe" and "I don't know". The scores were added and averages were obtained. The results were assessed according to the guidelines they reported to be following.

5. AB prophylaxis regimen: Open ended questions on the type of $A B$ prescribed (trade name or generic name), dosage and frequency of prescription and the route of administration.

The questionnaire was anonymous and a blank envelope was provided to each respondent to place his/her completed questionnaire into it. The envelope was sealed and handed to an investigator who was assigned to distribute and collect the questionnaires.

The data were analyzed using the Statistical Package for Social Services (SPSS) software package. Basic descriptive statistics and comparisons were done with the following variables: gender, age, year of graduation (years of experience in practice) and dental specialty. These variables were correlated to the responses obtained from the different cardiac conditions and the different types of dental procedures, inferential statistics were used to for controlling alpha error and the p-value was set at less than 0.05 .

Ethical approval and waiver of informed consent were obtained from the Ethical Committee of Taibah University, College of Dentistry.

\section{RESULTS}

Out of a total of 45 staff members, 39 responded ( $87 \%$ response rate) and there were more males (67\%) compared to females. The average age was 34.5 years (24-56; Std Dev. $10.4)$ and $46 \%$ had postgraduate qualifications. Almost half of the respondents (47\%) had more than 10 years experience and on average; postgraduate staff members had been qualified for 9.9 years (1-23; Std Dev. 7.2) with $41 \%$ having obtained their qualifications from Egypt and 12\% from India. Those without postgraduate qualifications had a maximum of 3 years experience. The breakdown according to gender and specialty is shown in Table $\mathbf{1 .}$

All except one respondent (97\%) reported to be using the AHA guidelines; therefore, the results were pooled and assessed according to these. More than two thirds (77\%) reported to "always refer" cardiac patients to their physician before undertaking invasive dental procedures while 66\% reported that their knowledge regarding the prevention of IE was based on previous training and reading scientific journals. Less than $15 \%$ stated that they acquired their knowledge from personal experience, attending seminars and participating in workshops.

The choice of prescribing ABs or not for certain cardiac conditions to prevent the onset of IE is shown in Table 2. There was no statistical difference between the responses in relation to the gender, years of experience or those with and without post graduate experience. The results were divided into two groups; common surgically treated heart conditions and congenital heart defects and the responses were "graded" according to the AHA guidelines [16]. The correct responses have been highlighted and the average of each category was calculated. For the common surgically treated cardiac conditions that require $\mathrm{AB}$ prophylaxis; almost two thirds (65\%) chose the correct answer. The congenital heart conditions, which according to the new guidelines do not require $\mathrm{ABs}$, less than a third $(28 \%)$ chose the correct option of not prescribing. On average $47 \%$ chose the correct option to either prescribe or not to prevent IE. Less than a third $(21 \%$ and $27 \%$ ) were unsure whether to prescribe $\mathrm{ABs}$ for common surgically treated conditions and the congenital heart conditions respectively.

Respondents were given 18 routine dental procedures and asked whether these procedures warranted the prescription of ABs to prevent IE. Table $\mathbf{3}$ shows the responses obtained and while there was no statistical difference between the responses when correlated with the number of years of experience or with the postgraduate qualifications, there were statistical differences between some of the responses when correlated to the gender $(\mathrm{p}<0.05)$. The highlighted columns are 
Table 1. Breakdown of Respondents According to Gender and Specialty $(\mathbf{N}=38)$

\begin{tabular}{|c|c|c|c|}
\hline \multirow{2}{*}{ Type of Specialty } & \multicolumn{2}{|c}{ Number } & \multirow{2}{*}{ Total (\%) } \\
\cline { 2 - 4 } & Males & 7 & $20(52)$ \\
\hline \hline No post graduate training & 13 & 2 & $4(10)$ \\
\hline Prosthodontist & 2 & 1 & $4(10)$ \\
\hline Endodontist & 3 & 2 & $3(8)$ \\
\hline Restorative Dentist & 2 & 0 & $3(8)$ \\
\hline Oral and Maxillo-Facial surgeon & 1 & 0 & $1(3)$ \\
\hline Oral Biologist & 1 & 0 & $1(3)$ \\
\hline Oral Medicine and Diagnosis & 1 & 0 & $1(3)$ \\
\hline Peadodontist & 1 & 13 & $1(3)$ \\
\hline Periodontist & 1 & 38 \\
\hline Total & 25 & 2 & 3 \\
\hline
\end{tabular}

Table 2. Responses Obtained Regarding the Prescription of Antibiotics for the Common Cardiac Conditions (N=38)

\begin{tabular}{|c|c|c|c|}
\hline \multirow{2}{*}{ Types of Cardiac Condition } & \multicolumn{3}{|c|}{ Responses in Percentage (\%) } \\
\hline & Yes & No & I don't know \\
\hline \multicolumn{4}{|l|}{ Common Surgically Treated Cardiac Conditions } \\
\hline Previous infective endocarditis & 100 & 0 & 0 \\
\hline Prosthetic cardiac valve & 94 & 3 & 3 \\
\hline Cardiac transplantation & 69 & 6 & 25 \\
\hline Recently placed coronary stents & 65 & 15 & 20 \\
\hline Intravascular pacemaker & 51 & 31 & 17 \\
\hline Unrepaired cyanotic heart disease & 31 & 20 & 49 \\
\hline Cardiac catherization without stents & 28 & 39 & 33 \\
\hline TOTAL & $65 \%$ & $14 \%$ & $21 \%$ \\
\hline \multicolumn{4}{|l|}{ Congenital heart defects } \\
\hline Hypertrophic cardiomyopathy & 29 & 37 & 34 \\
\hline Atrial septal defects & 34 & 34 & 31 \\
\hline Patent ductus arteriosis & 26 & 31 & 43 \\
\hline Ventricular septal defects & 46 & 26 & 29 \\
\hline Mitral valve prolapsed with regurgitation & 54 & 20 & 26 \\
\hline Rheumatic heart disease & 84 & 11 & 5 \\
\hline TOTAL & $45 \%$ & $28 \%$ & $27 \%$ \\
\hline
\end{tabular}

Table 3. The Prescription of Antibiotics for Dental Procedures in Relation to Gender $(\mathrm{N}=38)$

\begin{tabular}{|c|c|c|c|c|c|c|}
\hline Types of Dental Procedures & \multicolumn{6}{|c|}{ Responses (\%) } \\
\hline Surgical tooth extraction & 100 & 100 & 0 & 0 & 0 & 0 \\
\hline Periodontal surgery & 96 & 100 & 0 & 0 & 4 & 0 \\
\hline
\end{tabular}


Table 3. contd...

\begin{tabular}{|c|c|c|c|c|c|c|}
\hline \multirow{3}{*}{ Types of Dental Procedures } & \multicolumn{6}{|c|}{ Responses (\%) } \\
\hline & \multicolumn{2}{|c|}{ Yes } & \multicolumn{2}{|c|}{ No } & \multicolumn{2}{|c|}{ I don't know } \\
\hline & Males & Females & Males & Females & Males & Females \\
\hline Dental implants & 88 & 100 & 4 & 0 & 8 & 0 \\
\hline Biopsy & 88 & 100 & 0 & 0 & 12 & 0 \\
\hline Apicectomy & 88 & 100 & 4 & 0 & 8 & 0 \\
\hline Re-implantation of avulsed tooth & 81 & 100 & 4 & 0 & 15 & 0 \\
\hline Scaling and Polishing & 73 & 100 & 15 & 0 & 12 & 0 \\
\hline Simple tooth extraction & 88 & 85 & 12 & 15 & 0 & 0 \\
\hline Endodontic treatment & 56 & 84 & 44 & 7 & 0 & 7 \\
\hline Abscess drainage & 80 & 85 & 20 & 15 & 0 & 0 \\
\hline Dento-alveolar trauma & 83 & 92 & 13 & 0 & 4 & 8 \\
\hline Placement of fixed orthodontic bands & 12 & 46 & 65 & 39 & 23 & 15 \\
\hline TOTAL & \multicolumn{2}{|c|}{$84 \%$} & \multicolumn{2}{|c|}{$11 \%$} & \multicolumn{2}{|c|}{$5 \%$} \\
\hline Simple and complex restorations & $12 *$ & $69^{*}$ & $88^{*}$ & $31^{*}$ & 0 & 0 \\
\hline Tooth preparation and impression taking & $23 *$ & $85^{*}$ & $73 *$ & $15^{*}$ & 4 & 0 \\
\hline Placement of rubber dam & $17^{*}$ & $77^{*}$ & $71^{*}$ & $23^{*}$ & 12 & 0 \\
\hline Matrix band and wedging & $31 *$ & $92 *$ & $61 *$ & $8^{*}$ & 8 & 0 \\
\hline TOTAL & \multicolumn{2}{|c|}{$51 \%$} & \multicolumn{2}{|c|}{$46 \%$} & \multicolumn{2}{|c|}{$3 \%$} \\
\hline Intraligamentary local anaesthetic & 46 & 77 & 39 & 23 & 15 & 0 \\
\hline Inferior alveolar block & 46 & 61 & 50 & 39 & 4 & 0 \\
\hline
\end{tabular}

*statistically significant $(\mathrm{p}<0.05)$

the "correct" answers according to the AHA guidelines [16]. The majority (84\%) correctly chose to prescribe ABs for those dental procedures that required coverage. Of the dental procedures that did not require prophylactic $\mathrm{AB}$ coverage, only $46 \%$ correctly chose not to prescribe. In these procedures that did not justify the need for ABs, significantly more females chose to prescribe ABs than males. The two anaesthetic procedures (intraligamentary and inferior alveolar block) were not graded as the guidelines were not clear.

Almost all of the respondents (97\%) reported prescribing $2 \mathrm{~g}$ or $3 \mathrm{~g}$ of Penicillin and the majority of them $(90 \%)$ chose the oral route to administer the ABs. Almost two thirds (61\%) reported prescribing a single heavy dose and $29 \%$ prescribed multiple dosages (before and after treatment).

\section{DISCUSSION}

The relatively high response rate $(84 \%)$ could be attributed to the efforts of the senior academic staff in motivating staff members to complete the questionnaires and possibly due to the importance of this issue in the training and teaching of dental students in a community where the prevalence of cardiac conditions is relatively high [17].

More males had responded and this could be due to the fact that there were more males employed at the dental col- lege compared to females. The female dental college has only recently been opened (2010) and its staff complement is currently increasing. The average age of the respondents was 34 years, and this may be as a result of the presence of newly graduated (younger staff) and post graduate staff (relatively senior) members employed at the dental college. Almost half $(46 \%)$ had postgraduate qualifications; hence the dental college had an equal distribution of dentists and specialists teaching and supervising the students. Most postgraduate staff had qualified in Egypt (41\%) and India (12\%) as many of them were citizens of these countries.

Almost everyone (97\%) reported to be using the AHA guidelines and this was expected as all of the dental colleges in Saudi Arabia support the AHA guidelines. All foreign dentists wishing to work in Saudi Arabia must be registered by the Saudi Commission for Health Specialties and when participating in the entrance examinations must be familiar with these guidelines. As a result, many of them have applied these guidelines as these are accepted in Saudi Arabia. In other parts of the world, other guidelines such as BSAC and NICE are being taught and implemented. As these guidelines are constantly updated, it would be interesting to carry out a similar study and evaluate the knowledge amongst staff in these institutes. 
Many respondents $(78 \%)$ reported to "always" refer cardiac patients to their physician before undertaking invasive dental procedures. This is common practice and takes much of the responsibility off the dentist and places the responsibility onto the physician who must decide whether to prescribe or not. The Cochrane Review [1] concluded that ethically, dentists should consult with the patients and their physicians before deciding on whether to prescribe or not.

Two thirds of the staff $(66 \%)$ reported prescribing ABs based on previous training and reading scientific journals which was consistent with other studies [18].

In response to the different heart conditions, there were no statistically significant differences in the responses when correlated against the gender, age, specialty or years of experience and whether to prescribe or not. This was similar to results reported by Solomon et al. [12] who also showed no significant differences between these variables.

Two thirds of the respondents answered "correctly" for the surgically corrected cardiac conditions. This could be due to the guidelines having remained the same regarding the prescription of $\mathrm{ABs}$ for a cardiac condition where a foreign surgical intervention has been introduced.

However, for the congenital heart conditions, less than a third $(28 \%)$ chose the correct option of not prescribing. This could be due to the revision of the guidelines which currently suggest that congenital conditions no longer warrant the prescription of ABs. A large group of staff members $(21 \%$ and $27 \%$ ) was unsure whether to prescribe for the common and the congenital heart conditions respectively. On average $47 \%$ had correctly answered the questions which were better than the results reported by Nelson [14] (33\%) and Murrah [15] $(35 \%)$. However, it must be noted that the types of cardiac conditions that were used in these studies are not listed and these comparisons need to be interpreted with caution. The variation in responses was not unexpected as other studies have also shown a wide variation even amongst medical and dental specialists [14].

The responses regarding whether ABs should be prescribed or not for different dental procedures were also fairly equally distributed. The majority (84\%) reported correctly prescribing $\mathrm{ABs}$ for those procedures which needed coverage. However, many were overprescribing for conditions that did not require ABs. A possible reason could be that staff was unsure and decided to err on the side of caution and rather overprescribe. This was also recommended by Cunha et al. [7] who reported on a patient that did not receive prophylactic coverage and went on to develop complications following dental treatment. However, this was a single case and it was not mentioned which dental procedure had been performed.

A review by Roberts [19] proposed that "everyday" oral bacteria could be more responsible for IE than dental procedures. Seymour et al. [20] reported that the prevalence of bacteraemia varied according to different dental procedures with multiple extractions being responsible for between $68 \%$ and $100 \%$ of the bacteria in the mouth. Other procedures such as scaling and root planning and periodontal surgery accounted for $80 \%$ and $88 \%$ of bacteria respectively and patients undergoing these types of treatment should be covered with the necessary ABs. Although there has been only one suspected case of a patient developing IE following dental treatment, [7] it is not possible both ethically and practically to carry out such research. Therefore, the guidelines are gathered mostly from experimental animal models, bacterial susceptibility studies and clinical experience [21]. Another case control study [21] supported the use of prophylaxis coverage in patients with high risk reporting an odds ratio of 0.09 , which indicated a $91 \%$ protective efficacy. Hence for the safety of both dentists and patients, they recommended the use of an acceptable and standardized guideline.

There was a statistical difference in the prophylactic prescription between males and females for four dental procedures: placement of a rubber dam, tooth preparation and impression taking, matrix band and wedging and simple and complex restorations. In each of these procedures, females reported to prescribe more frequently compared to males, even though the latest AHA guidelines do not recommend prescribing for these procedures. The reasons could be that the guidelines do not describe specific dental procedures and hence there may be variation depending on their interpretation [4]. In addition, if there is gingival manipulation, such as with rubber dam placement or matrix band placement, then according to the AHA guidelines, one could prescribe ABs. Therefore, depending on the individual situation and the oral hygiene of the patient, practitioners could justify the prescription of ABs for "low risk" dental procedures.

Almost half of the females (46\%) stated they would prescribe $\mathrm{ABs}$ for orthodontic bands while $65 \%$ of the males responded in the negative. The AHA guidelines state that the initial placement of the bands requires coverage while the routine placement of brackets does not. It is possible because some respondents did not know there was a difference between the bands and the brackets and hence the varied results for this option.

More than half of the males chose "I don't know" for many procedures. This showed the uncertainty and confusion that existed when prescribing ABs. This was similar to results reported by Chate, [4] who concluded that clinical treatment is compromised when there are conflicting guidelines which exist for the same condition.

Almost everyone reported to prescribe the drug of choice, Penicillin $(2 \mathrm{~g}$ or $3 \mathrm{~g})$. The previous guidelines recommended $3 \mathrm{~g}$ whilst the updated guidelines recommend $2 \mathrm{~g}$. Hence this could be responsible for the variation in the dose. This was much higher than another study [12] which reported that more than half of the respondents did not prescribe $\mathrm{ABs}$ in accordance with the latest AHA guidelines. This difference could be attributed to the fact that this study was conducted in 1990, and since then dentists have become more aware of the prescribed guidelines. The majority reported to administer the drug orally as this is usually the route of administration. More than half chose to offer a single dose and this is consistent with the latest guidelines. The remaining respondents, who chose to administer multiple doses (before and after treatment), could be doing so as a result of the old guidelines which recommended multiple doses.

The wide variation in the results is of concern to the dental college. It is expected that most dental colleges in Saudi Arabia would have similar results as reported by many inter- 
national studies. As a result, there have been plans to collaborate with other dental colleges and carry out similar studies to confirm whether this problem is as widespread as anticipated. If so, then nationally standardized guidelines should be discussed and implemented across the Kingdom to ensure that all students and patients receive the most appropriate and acceptable treatment modalities.

\section{RECOMMENDATIONS}

It is recommended to update staff members on the latest AHA guidelines regularly and continuously. These guidelines should be formally included in the student's curriculum and they must be assessed on its contents. Additionally, the guidelines should be placed in strategic positions throughout the dental clinics. This would ensure that students and staff are constantly exposed to them and can refer to them when in doubt as to whether to prescribe or not. It is also recommended to discuss these results with other dental colleges so that a standardized guideline can be formulated for teaching institutions for both medical and dental students in the Kingdom of Saudi Arabia. It is further emphasized that a similar study be conducted amongst private dentists in and around Medina. Following on the results, workshops could be initiated for the updating of current and acceptable guidelines for the prevention of IE.

\section{CONCLUSION}

Although most staff reported using the AHA guidelines, many were not following them correctly. A large proportion was unsure about whether to prescribe or not for different cardiac conditions. There was also confusion about the different dental procedures that may or may not require prophylaxis. This could be due to the regular and drastic changes in the guidelines and the relatively vague statements regarding the prescription of $\mathrm{ABs}$ for specific dental procedures. It is essential to continuously update and train staff members and private practitioners on the latest guidelines to ensure that patients and students receive the best treatment and advice.

\section{CONFLICT OF INTEREST}

The authors confirm that this article content has no conflicts of interest.

\section{ACKNOWLEDGEMENTS}

The authors would like to thank Dr M Kaaki for his invaluable assistance in the distribution and collection of the questionnaires.

\section{REFERENCES}

[1] Oliver R, Roberts GJ, Hooper L, Worthington HV. Antibiotics for the prophylaxis of bacterial endocarditis in dentistry. Cochrane Database Syst Rev 2008; 8(4): CD003813.

[2] Gopalakrishnan PP, Shukla SK, Tak T. Infective endocarditis: rationale for revised guidelines for antibiotic prophylaxis. Clin Med Res 2009; 7(3): 63-8.
[3] Allen U. Infective endocarditis: updated guidelines. Paediatr Child Health 2010; 15(4): 205-8.

[4] Chate RA. An infective endocarditis audit illustrates why dental guidelines in general need to be kept clear, simple and unambiguous. Br Dent J 2008; 205(6): 331-5.

[5] Nakano K, Ooshima T. Common knowledge regarding prevention of infective endocarditis among general dentists in Japan. J Cardiol 2011; 57(1): 123-30.

[6] Parrish A, Maharaj B. Prevention of infective endocarditis in developing countries-justifiable caution? S Afr Med J 2012; 102(8): $652-4$.

[7] Cunha BA, D'Elia AA, Pawar N, Schoch P. Viridans streptococcal (Streptococcus intermedius) mitral valve subacute bacterial endocarditis (SBE) in a patient with mitral valve prolapse after a dental procedure: The importance of antibiotic prophylaxis. Heart Lung 2010; 39(1): 64-72.

[8] Coutinho AC, Castro GF, Maia LC. Knowledge and practices of dentists in preventing infective endocarditis in children. Spec Care Dentist 2009; 29(4): 175-8.

[9] Doshi D, Baldava P, Reddy S, Singh R. Self-reported knowledge and practice of American Heart Association 2007 guidelines for prevention of infective endocarditis: a survey among dentists in Hyderabad City, India. Oral Health Prev Dent 2011; 9(4): 347-51.

[10] Zadik Y, Findler M, Livne S, Levin L, Elad S. Dentists' knowledge and implementation of the 2007 American Heart Association guidelines for prevention of infective endocarditis. Oral Surg Oral Med Oral Pathol Oral Radiol Endod 2008; 106(6): e16-9.

[11] Wilson W, Taubert KA, Gewitz M, et al. Prevention of infective endocarditis: guidelines from the American Heart Association: a guideline: Am Heart Associat Rheumatic Fever. Circulation 2007; 116: $1736-54$.

[12] Solomon M, Raveh D, Schlesinger Y, Yinnon AM. Assessment of knowledge of guidelines for the prevention of infective endocarditis amongst clinicians in a teaching hospital. J Hosp Infect 2000; 45(4): 311-7.

[13] Gutschik E, Lippert S. Dental procedures and endocarditis prophylaxis: experiences from 108 dental practices. Scand J Dent Res 1990; 98(2): 144-8.

[14] Nelson CL, van Blaricum CS. Physician and dentist compliance with American Heart Association guidelines for the prevention of bacterial endocarditis. J Am Dent Assoc 1989; 118(2): 169-73.

[15] Murrah VA, Merry JW, Little JW, Jaspers MT. Compliance with guidelines for management of dental school patients susceptible to infective endocarditis. J Dent Educ 1987; 51(5): 229-32.

[16] Farbod F, Kanaan H, Farbod J. Infective endocarditis and antibiotic prophylaxis prior to dental/oral procedures; latest revision to the guidelines by the American Heart Association published April 2007. Int J Oral Maxillofac Surg 2009; 38(6): 626-31.

[17] Alabdulgader AAA. Congenital heart disease in Saudi Arabia: current epidemiology and future projections. East Mediterr Health J 2006; 2(2): 157-67.

[18] Abukaraky AE, Abu Afifeh K, Khatib AA, et al. Antibiotics prescribing practices in oral implantology among Jordanian dentists. A cross sectional, observational study. BMC Res Notes 2011; 4: 26670 .

[19] Roberts G. Dentists are innocent! "Everyday" bacteraemia is the real culprit: A review and assessment of the evidence that dental surgical procedures are a principal cause of bacterial endocarditis in children. Pediatr Cardiol 1999: 20: 317-25.

[20] Seymour RA, Lowry J, Whitworth JM, Martin MV. Infective endocarditis, dentistry and antibiotic prophylaxis; time for a rethink? Br Dent J 2000; 189(11): 610-6.

[21] Imperiale TF, Horwitz RI. Does prophylaxis prevent postdental infective endocarditis? a controlled evaluation of protective efficacy. Am J Med 1990; 88(2): 131-6.

Received: March 19, 2013

Revised: April 24, 2013

Accepted: April 30, 2013

(C) Bhayat et al.; Licensee Bentham Open.

This is an open access article licensed under the terms of the Creative Commons Attribution Non-Commercial License (http://creativecommons.org/licenses/by-nc/3.0/) which permits unrestricted, non-commercial use, distribution and reproduction in any medium, provided the work is properly cited. 